\title{
Proinflammatory Pathways Engaged by Oral Pathogen Porphyromonas gingivalis in Upregulation of Matrix Metalloproteinase-9 Expression in Periodontal Disease
}

\author{
Bronislaw L. Slomiany, Amalia Slomiany \\ Research Center, Rutgers School of Dental Medicine, Rutgers, The State University of New Jersey, Newark, USA \\ Email: slomiabr@sdm.rutgers.edu
}

How to cite this paper: Slomiany, B.L. and Slomiany, A. (2018) Proinflammatory Pathways Engaged by Oral Pathogen Porphyromonas gingivalis in Upregulation of Matrix Metalloproteinase-9 Expression in Periodontal Disease. Journal of Biosciences and Medicines, 6, 77-94.

https://doi.org/10.4236/jbm.2018.66006

Received: May 20, 2018

Accepted: June 22, 2018

Published: June 25, 2018

Copyright (c) 2018 by authors and Scientific Research Publishing Inc.

This work is licensed under the Creative

Commons Attribution International

License (CC BY 4.0).

http://creativecommons.org/licenses/by/4.0/

\begin{abstract}
Matrix metalloproteinase-9 (MMP-9) is a potent endopeptidase implicated in a wide range of inflammatory and neoplastic diseases, including chronic periodontitis, a persistent oral mucosal inflammation attributed primarily to infection by $P$. gingivalis. Here, we review the signaling pathways engaged by $P$. gingivalis in controlling the processing and secretion of MMP-9. The induction in oral mucosal expression of MMP-9 by $P$. gingivalis relays primarily on its key endotoxin, LPS, engagement of TLR4 and the activation of MAPK, ERK and p38 cascades implicated in the stimulation of Rac1 and $\mathrm{CPLA}_{2}$. The ERK-mediated $\mathrm{CPLA}_{2}$ phosphorylation plays an essential role in its membrane translocation with Rac1, while p38 localization with Rac1 promotes $\mathrm{CPLA}_{2}$ activation and the induction in MMP-9. Moreover, the induction in MMP-9 secretion by the LPS and the modulatory influence of peptide hormone, ghrelin, occur at the level of MMP-9 processing between ER and Golgi, with the involvement of factors that control secretory cargo sorting, Arf1 GTPase and PKD2. The secretion of MMP-9, furthermore, occurs in concert with the changes in stability dynamics of microtubules (MTs), which affect the Golgi localization of Arf1 and the recruitment and activation of PKD2. The induction in MMP-9 secretion by LPS is accompanied by the enhancement in MT stabilization and $\alpha$-tubulin phosphorylation on Ser, while the MT destabilization associated with the modulatory influence of ghrelin, is manifested by $\alpha$-tubulin phosphorylation on Tyr. Thus, the factors capable of affecting MT dynamics and MMP-9 secretion present a tempting target for the therapeutic intervention in the treatment of chronic periodontitis.
\end{abstract}

\section{Keywords}

P. gingivalis, Periodontal Disease, Inflammatory Response, LPS, 


\section{Introduction}

Porphyromonas gingivalis, a rod-shaped, Gram-negative anaerobe together with two other oral bacterial species colonizing the oral cavity, Treponema denticola, and Tannerella forsythia, form so called "red bacterial complex" recognized as the primary etiological cause of periodontitis, a chronic inflammatory disease that leads to progressive destruction of teeth-supporting tissue and is the major cause of adult tooth loss [1] [2] [3] [4]. A growing body of evidence, moreover, indicates that periodontitis affects the course and pathogenesis of a number of systemic diseases such as cardiovascular disease, infective endocarditis, atherosclerosis, bacterial pneumonia, diabetes mellitus, leukemia, and low birth weight [5] [6] [7]. There are also strong supporting data linking periodontitis and $P$. gingivalis infection to rheumatoid arthritis, and the progression and metastasis of oral squamous cell carcinoma [8] [9] [10] [11].

Indeed, $P$. gingivalis, by far the most prominent member of the bacterial flora found in periodontal packets of people with gum disease [12], has to its disposal an impressive array of virulence factors that provoke the inflammatory response of the host tissue [13] [14]. These include its cell surface major (FimA) and minor (Mfal) fimbriae proteins, elaborated arginine and lysine specific cysteine proteinases, referred to as gingipain $R$ and gingipain $K$ [15] [16], collagenases, capable of extracellular matrix proteins degradation and activation of the host matrix metalloproteinases [17] [18] [19], and the secreted sufatases directed towards cell membrane glycosphingolipids as well as proteoglycans of extracellular matrix [3] [20]. However, the virulence factor of particular significance to the pathogenic action of $P$. gingivalis leading to the development of periodontitis is its cell-wall lipopolysaccharide (LPS) [21] [22].

In general, the bacterial LPS consists of membrane anchored hydrophobic domain, referred to as lipid A, non-repeating core oligosaccharide region, and a distal polysaccharide structure referred to as $\mathrm{O}$-antigen chain. The lipid A region is composed of phosphorylated $(1,6)$-linked diglucosamine backbone with four to seven fatty acyl chains ranging from 10 to 16 carbon units attached to it. Of these, four acyl chains are directly linked to the glucosamine backbone, and the remaining being attached to the hydroxyl groups of the lipid chains [23] [24]. The non-repeating core oligosaccharide region is rich in heptose and KDO (keto-deoxyoctulosonate), and coupled to the O-specific highly heterogeneous set of carbohydrate chains extending from the cell surface [23] [24] [25]. The endotoxin and the immunological properties of LPS reside mainly in the heterogeneous acylation character of lipid A domain, which in different bacterial species shows considerable variation in the acylation pattern and the number of phosphate groups, while the core and O-specific regions of LPS appear to have only minor role in the immune recognition [23] [24] [26] [27] [28]. 
Investigations into the events associated with oral mucosal responses to $P$. gingivalis LPS demonstrated a marked increase in epithelial cell apoptosis and proinflammatory cytokine expression, up-regulation in endothelin-1 (ET-1) and TNF- $\alpha$ release, excessive nitric oxide (NO) and prostaglandin (PGE2) generation, and the elevated levels of matrix metalloproteinase-9 (MMP-9) production [18] [21] [29] [30] [31] [32] [33]. Hence, considering that the elevated levels of MMP-9, along with the increased proinflammatory cytokine production are implicated in pathogenesis of chronic periodontitis, in this article we review data on the signaling pathways engaged by periodontopathogen, $P$. gingivalis, in controlling the processing and secretion of MMP-9.

\section{2. $P$. gingivalis and TLR4 Activation}

\subsection{MMP-9 in Inflammatory Responses to Bacterial LPS}

The mechanism underlying the inflammatory processes elicited by $P$. gingivalis in oral mucosa relies on the interaction of its LPS with Toll-like receptor-4 (TLR4). The LPS triggered stimulation of TLR4 signaling pathways (Figure 1) leads to activation of $\mathrm{I} \kappa \mathrm{B}$-kinase complex (IKK) and up-regulation in mitogen-activated protein kinases (MAPKs), which trigger the activation of transcriptional factors that exert control over a wide range of proinflammatory mediators, including MMP-9 [32] [33] [34].

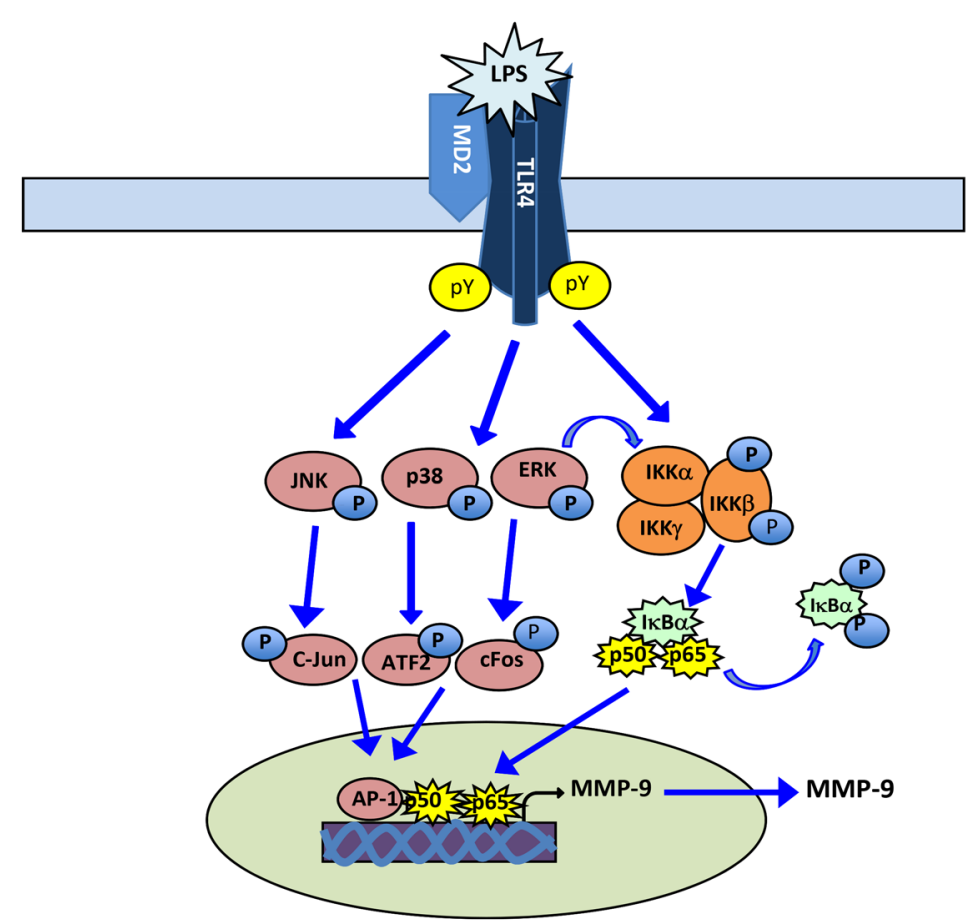

Figure 1. Signaling path ways and transcription factors involved in oral mucosal secretion of MMP-9 in responses to $P$. gingivalis LPS. Ligation of TLR4 by the LPS results in the activation of IKK complex and MAP kinases, ERK, p38 and JNK, and triggers the nuclear translocation of transcription factors, AP-1 and NF- $\kappa \mathrm{B}$, involved in the induction of MMP-9 gene transcription. $\mathrm{pY}$, phosphotyrosine; $\mathrm{P}$, phosphate. 
Matrix metalloproteinases (MMPs) are a family of zinc-dependent endopeptidases implicated in the remodeling of extracellular matrix under normal physiological conditions, as well as during angiogenesis, wound healing, tumor metastasis, and a wide range of degenerative and inflammatory diseases, including oral cancer, Sjogren's syndrome, rheumatoid arthritis, oral lichen planus, and chronic periodontitis [3] [19] [34]-[39]. This multidomain enzymes are composed of the active site and the catalytic metal binding domain which is kept inactive by $\mathrm{N}$-terminal pro-peptide domain (34). The catalytic domain contains the zinc binding site, a calcium ion and conserved methionine. These are essential for maintenance of MMP three-dimensional structure and enzyme activity. The unique amino acid sequences within the active site are of importance for the enhancing of catalytic efficiency towards gelatin and peptide substrates [34]. The pro-peptide domains of MMPs contain the cysteine switch sequence, which ligates the catalytic zinc to maintain the enzyme inactive. Activation of the enzyme requires either proteolytic processing of the pro-peptide domain or steric disruption of the pro-peptide conformation [34].

While the main function of MMPs is the regulation of cell matrix composition, the enhanced production of MMPs, and MMP-9 in particular, is associated with host reaction to microbial and fungal infections [40] [41], as well as characterizes inflammatory response to lipopolysaccharide (LPS) of Gram-negative bacteria in several different cell systems [25] [42] [43] [44]. The elevated levels of MMP-9, elicited in oral mucosa in response to Porphyromonas gingivalis and its key endotoxin, LPS, furthermore, are directly responsible for persistent mucosal inflammation that leads to periodontal lesions and progressive destruction of teeth-supporting tissue, including bone loss [4] [19] [38] [41] [44]. There are also strong indications that enhanced levels of MMP-9 in serum of periodontal disease patients may contribute to extra-oral sequela, such as a decrease in cardiac function and cardiovascular disease [3] [4] [17]. Interestingly, the association between elevated serum content of MMP-9 and the increased risk of cardiovascular disease was also observed in patients with $H$. pylori-associated gastritisdiseases, and a decrease in the mucosal level of MMP-9 occurred following successful $H$. pylori eradication therapy [45] [46]. There are strong indications that the elevated levels of MMP-9 contribute directly to the inflammatory process by proteolytic tissue destruction, recruitment of inflammatory cells, and the generation of inflammatory signals. Indeed, the induction in MMP-9 expression is observed in wound healing, whereas the inhibition of MMP-9 has been linked to aberrant re-epithelization [9] [34] [35]. Moreover, MMPs, including MMP-9, are involved in extracellular matrix and basement membrane degradation and the activation of factors that perpetuate the inflammatory process [35].

Although the rate-limiting step in MMP-9 regulation is gene transcription, the expression and activity of this heavily glycosylated, $92 \mathrm{kDa}$, endopeptidase remains also under the influence of posttranslational processing, proenzyme activation, and the inhibition by the family of endogenous tissue inhibitors of me- 
talloproteinases (TIMPs) [34] [42]. This multifaceted control assures the low MMP-9 expression in normal tissue, and its rapid release and activation in response to inflammatory stimulus such as LPS [43]. Therefore, therapies aimed at decreasing the MMP-9 expression and its enzymatic activity by the use of specific MMP inhibitors could provide much needed options in the treatment of inflammatory diseases, including chronic periodontitis. A positive development along these lines is the successful use of modified tetracycline derivative in the treatment of periodontitis [34].

\subsection{LPS-Induced TLR4 Activation and MMP-9 Expression}

Studies into the processing of MMP-9 in response to LPS stimulation indicate that MMP-9 expression is tightly controlled at the transcriptional level by MAPK cascade, including ERK, JNK, and p38, which along with IKK exert their influence over transcriptional factors AP-1 and NF-kB activation [47] [48]. Moreover, these responses to $P$. gingivalis are mediated through the LPS triggered TLR4 activation [18] [33].

Indeed, the available data, suggests that MAPK cascade activation upon LPS stimulation plays an important role in the regulation of intracellular trafficking, membrane translocation, and the release of secretory products [43] [49] [50]. The LPS-induced p38 activation and its recruitment to the cytosolic aspect of the membrane-localized Rac1 GTPase has been linked to disintegrin-metalloprotease ADAM17 activation [32] [49], and the up-regulation in MAPK and Rac activation was observed in association with LPS-induced pulmonary inflammation and TLR4-stimulated phagocytosis [51] [52]. There are also strong indications that ERK activation upon LPS stimulation plays an important role in the phosphorylation of cytosolic phospholipase $\mathrm{A}_{2}\left(\mathrm{CPLA}_{2}\right)$ that facilitates the enzyme translocation from cytosol to membrane to gain access to phospholipid substrates [33] [50]. Moreover, further up-regulation in $\mathrm{CPLA}_{2}$ activation has been reported to occur with the involvement of Rac1/p38 complex [53] [54]. Consistent with these facts are the data pointing to the role of MAPK signaling in targeting of $\mathrm{cPLA}_{2}$ to intracellular membranes involved in secretory cargo processing, such as the Golgi vesicle formation and the membrane-vesicle fusion events that affect the secretory cargo release [53] [55] [56].

Therefore, taking into account the evidence that MMP-9 undergoes extensive processing in the Golgi network and the fact that oral mucosal inflammatory responses to $P$. gingivalis are characterized by the rapid rise in MMP- 9 release, we investigated the nature of factors involved in the processing of signaling cascade initiated by the LPS [18]. As summarized in Figure 2, our findings demonstrated that $P$. gingivalis LPS-elicited induction in the salivary gland acinar cell MMP-9 release is associated with MAPK, ERK and p38 activation, and occurs with the involvement of Rac1 and CPLA ${ }_{2}$ ). Moreover, the release of MMP-9 involves ERK-mediated phosphorylation of $\mathrm{CPLA}_{2}$ on $\mathrm{Ser}^{505}$ that is essential for its membrane translocation with Rac1, and that this process requires $\mathrm{p} 38$ activation. Apparently, the activation and membrane localization of p38 with Rac1-GTP 


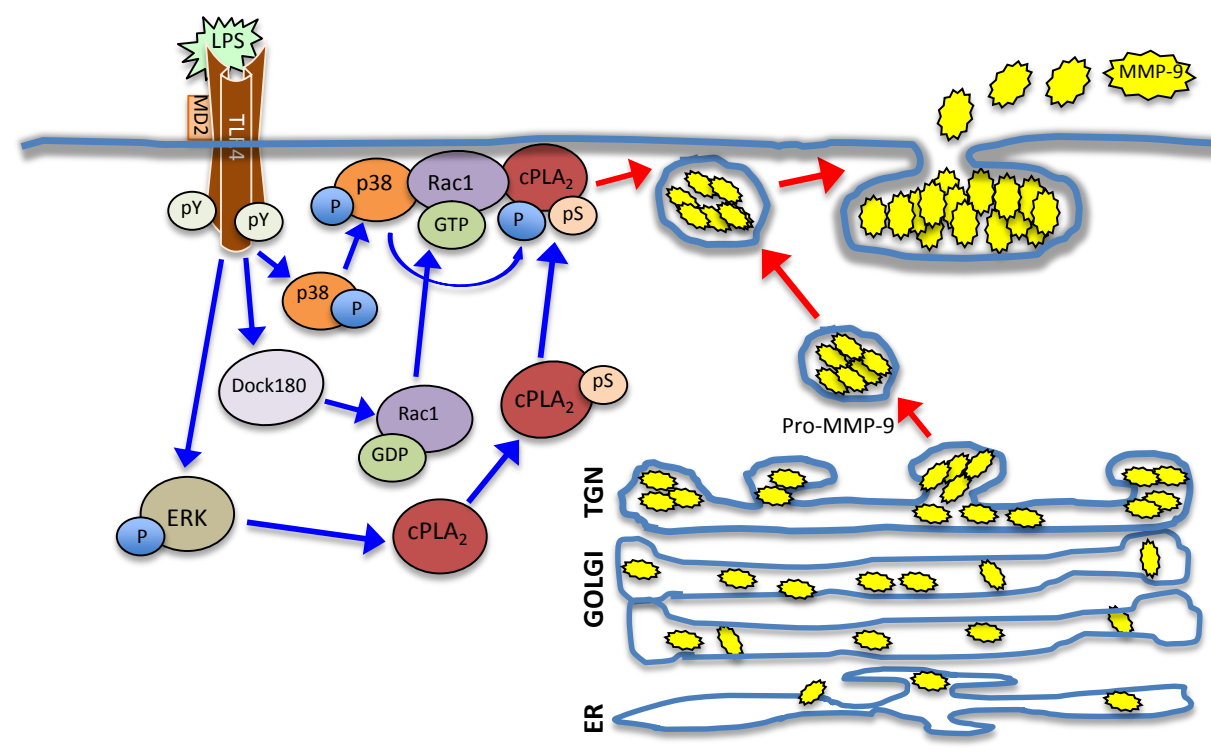

Figure 2. Diagram of the pathways involved in $P$. gingivalis LPS-elicited induction in salivary gland acinar cell MMP-9 release. Binding of the LPS to TLR4 triggers up-regulation in ERK and p38 MAPK activation, as well as induces the pathway of GEF, Dock180-mediated GTP-Rac1 formation and its membrane translocation. This in turn, promotes the membrane localization of pp38 with the Rac1-GTP, while pERK is involved in the phosphorylation of $\mathrm{CPLA}_{2}$ on $\mathrm{Ser}^{505}$, which prompts its recruitment to the $\mathrm{Rac1} / \mathrm{p} 38$ complex, and the induction in $\mathrm{CPLA}_{2}$ activation. The $\mathrm{CPLA}_{2}$, by acting on membrane phospholipids, affects the membrane fusion events, and leads to the induction in MMP-9 release. P phosphate, pS phosphoserine, pY phosphotyrosine.

plays a pivotal role in $\mathrm{CPLA}_{2}$-dependent induction in MMP-9 release [18]. Collectively, the findings infer that $P$. gingivalis LPS-induced up-regulation in the acinar cell MMP-9 release requires ERK-dependent recruitment of $\mathrm{CPLA}_{2}$ to the membrane localized Rac1/p38 complex.

\section{Factors Affecting MMP-9 Processing}

\subsection{ADP-Ribosylation Factors}

MMP-9 is a highly glycosylated endopeptidase, consisting of both $\mathrm{N}$ - and O-linked carbohydrate chains, and hence its processing along the secretory pathway relies heavily on the co- and posttranslational modifications acquired during the protein transit between the endoplasmic reticulum (ER), Golgi, and trans-Golgi network (TGN) [34] [57]. Like secretion of other salivary proteins and glycoproteins, the trafficking of MMP-9 cargo remains under a strict control of factors that affect the membrane recruitment and activation of various coat and cargo proteins [58] [59] [60].

Of particular significance to the secretory cargo trafficking and sorting through Golgi-trans-Golgi (TGN) is the role of small GTP-binding proteins of the ADP-ribosylation factor (Arf) family [60] [61]. Arfs are small $20 \mathrm{kDa}$ GDP/GTP-binding proteins belonging to the Ras superfamily of GTPases that control protein transport and recycling between different cellular compartments, and cytoskeleton remodeling [60]. The Arfs are active when bound to GTP and inactive when bound to GDP, and the activation status is controlled by the gua- 
nine nucleotide exchange factors (GEFs) [61] [62]. All six members of the Arf family are characterized by presence the N-terminal myristoylate tail, which allows their association with membranes, and based on their amino acid sequence homology are grouped into three classes. Of these, class I and class II Arfs are associated with the Golgi apparatus and implicated in the regulation of cell secretory function, while class III Arf, Arf6, functions in the endocytic system [59] [63]. While inactive Arfs are cytosolic, the stimulus activated GTP-bound class I Arfs (Arf1, Arf2, and Arf3) rapidly translocate to the Golgi membrane compartments and assume the principal role in the recruitment of various cytosolic coat and cargo adaptor proteins, exchange factors, and lipid modifying enzymes, including phospholipase $\mathrm{D}$, phosphatidylinositol 4-kinase and protein kinase $\mathrm{D}$ (PKD), that are essential for regulation of ER-to-Golgi traffic [58] [60] [63] [64].

\subsection{Protein Kinase D}

PKD is a family of serine/threonine kinases consisting of three closely related calmodulin-dependent members (PKD1, PKD2, and PKD3), that play a crucial role in the regulation of Golgi structure and function by phosphorylation of the TGN-localized substrates required for subsequent shedding of cargo-containing vesicles [58] [65]. Upon stimulation, the members of PKD family are first recruited from the cytosol to the diacylglycerol-rich membrane environment, including the Golgi complex, via interaction with Arf-GTP and then undergo activation by PKC-mediated transphosphorylation of their activation loop on $\mathrm{Ser}^{744}$, followed by autophosphorylation on $\mathrm{Ser}^{748}$ [58] [66]. Therefore, PKD along with PKC form a key convergence and integration node for signals triggered by Toll-like receptor (TLR) activation as well as those arising through G protein-coupled receptor (GPCR) stimulation.

Thus, it is becoming apparent that $\mathrm{PKD} / \mathrm{PKC}$ axis is not only the signaling target of LPS-induced TLR4 activation that defines the extent of inflammatory response, but also plays a major role in modulation of inflammation by a peptide hormone, ghrelin [67] [68] [69]. This endogenous ligand for the growth hormone secretagoguereceptor type 1a (GHS-R1a), initially isolated from the stomach, and later identified in saliva and the acinar cells of salivary glands, has emerged recently as a principal modulator of the local inflammatory responses to bacterial infection, including that to $P$. gingivalis and $H$. pylori [25] [70] [71] [72]. Remarkably, the anti-inflammatory effect of ghrelin observed during progressive heart failure, associated with periodontal disease, has been linked to the suppression in MMP-9 expression [3] [4] [73]. Moreover, we revealed that $P$. gingivalis LPS-elicited induction in MMP-9 secretion and the modulatory influence of ghrelin occur at the level of MMP-9 processing between the endoplasmic reticulum (ER) and Golgi [74], and is associated with GEF-mediated Arf1 activation and the TGN recruitment of PKD2, followed by its activation by phosphorylation on Ser by the $\operatorname{PKC} \delta$. Whereas the modulatory effect of ghrelin is manifested by the SFK-PTKs-dependent phosphorylation of PKD2 on Tyr 
(Figure 3). Hence, Arf1/PKD2 junction could represent a novel target for development of more effective treatments of periodontal disease.

\section{Changes in Microtubule Dynamics and MMP-9}

Investigations into the processing of MMP-9 along the ER, Golgi and TGN indicate that, in addition to factors that affect the membrane recruitment and activation of various coat and payload proteins, the trafficking of MMP-9 cargo occurs in concert with the changes in stability dynamics of the major cytoskeleton polymeric structures, microtubules (MTs) [56] [75] [76] [77]. Indeed, the literature data provide an ample evidence that cytoskeleton plays an important role not only in cell division and motility, but also is involved in the intracellular transport and positioning of organelles and vesicles, and the MTs have been directly implicated in the regulation of MMP-9 secretion in several different cell systems [56] [76] [78] [79]. The MTs polymers, assembled from heterodimers of $\alpha$ - and $\beta$-tubulin proteins in a head-to-tail manner, form a dense network of

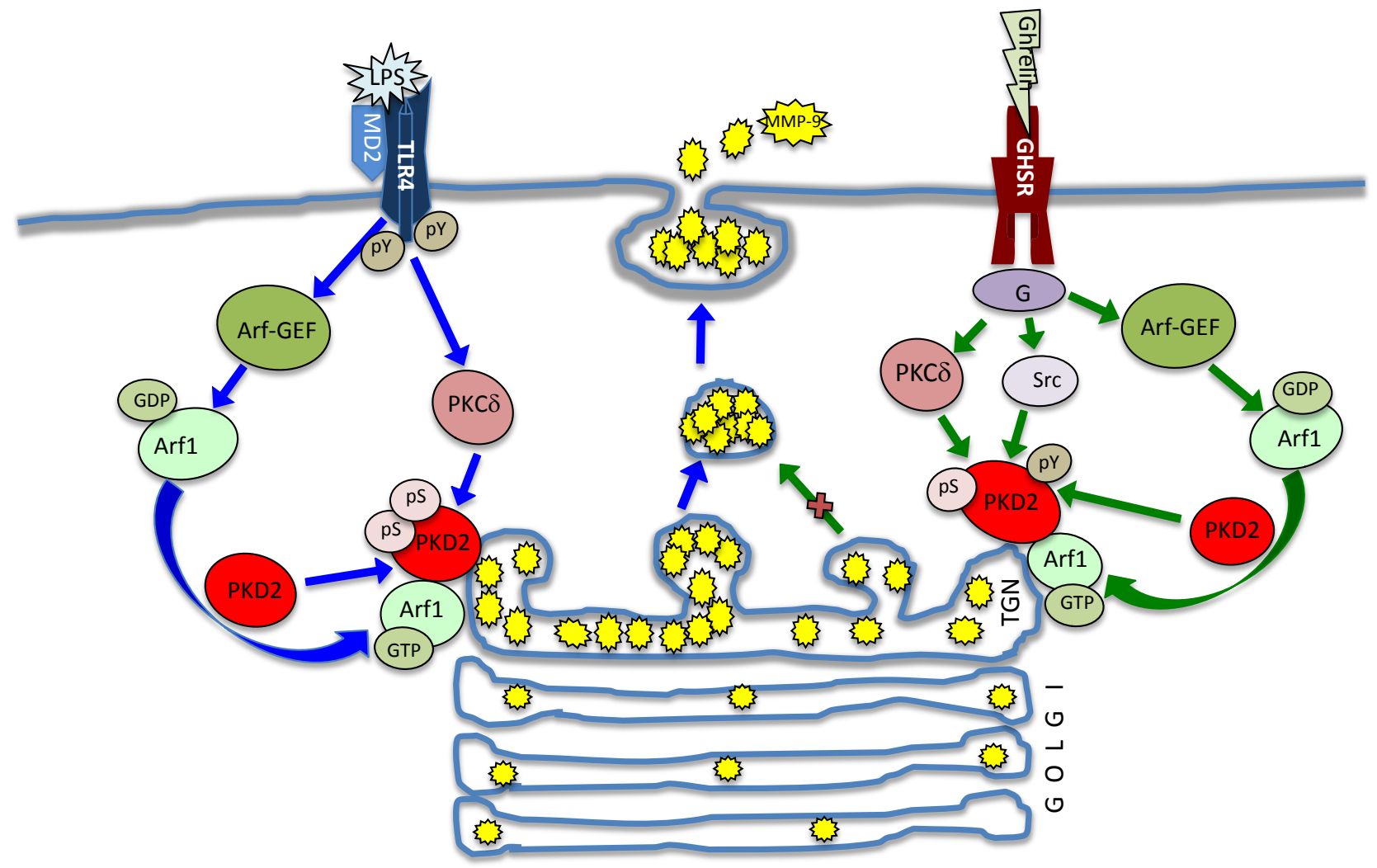

Figure 3. Schematic representation of the role of Arf1/PKD2 in mediation of salivary gland acinar cell MMP-9 secretion in response to $P$. gingivalis LPS stimulation, and the modulatory influence of ghrelin. Engagement of TLR4 by the LPS triggers Arf-GEF-mediated Arf activation and its translocation to the Golgi. This promotes the localization of PKD2 with Arf1-GTP at the TGN, and its activation by phosphorylation on Ser by the PKC $\delta$. The activated PKD2, by acting on the TGN-localized substrates, affects the vesicle fission events and thus enhances the secretion of MMP-9. Ligation by ghrelin of GHS-R1a, on the other hand, leads to Arf-GEF-mediated Arf1 activation and PKD2-to-TGN localization, followed by the SFK-PTKs-mediated phosphorylation of PKD2 on Tyr that maintains the PKD2 activity at its normal regulatory level. G heterotrimeric G protein, pS phosphoserine, $\mathrm{pY}$ phosphotyrosine. 
filamentous tubes that undergo frequent alternating periods of growth (polymerization) and shortening (depolymerization) termed "dynamic instability" [77] [78]. Interestingly, the changes in tumor cell stability dynamics are associated with malignant transformation, and the enhancement in MT stabilization along with the increase in MMP-9 production has been observed during inflammation and wound healing [34] [80].

The diverse aspects of MT dynamics arise from accumulation of a variety of posttranslational modifications on the tubulin subunits, including acetylation, detyrosination, polyglutamylation, polyglycylation and phosphorylation, and are further tuned and refined through binding of MT-associated proteins [78] [81] [82] [83]. The changes in MT stability dynamics and the increase in $\alpha$-tubulin acetylation have been directly linked to the increase in MMP-9 secretion elicited by MT stabilizing agents [56], and we have shown recently that MT destabilizing agents that inhibit microtubule polymerization exert the inhibitory effect on the increase in salivary gland acinar cell MMP-9 secretion elicited by $P$. gingivalis LPS [74]. There are also reports pointing to the role of tubulin phosphorylation in MT stabilization, and the evidence suggests the involvement of PKC and Src family tyrosine kinase (SFK) in this process [80] [84] [85]. The role of tubulin phosphorylation in MT stabilization is furthermore, supported the results indicating that phosphorylation of $\alpha$-tubulin on Ser prolongs the duration of MT growth, while the phosphorylation on Tyr promotes MT dynamic instability [80] [84] [85].

Therefore, considering that $\mathrm{PKC}$ and SFKs play a major role in propagation of oral mucosal inflammatory response to $P$. gingivalis associated with the enhancement in MMP-9 production, as well as are the targets of modulatory influence of a peptide hormone, ghrelin [33] [74] [86], we assessed the influence of $P$. gingivalis LPS and ghrelin on tubulin phosphorylation in salivary gland acinar cells and the MMP-9 secretory processes affected by the changes in MT dynamics [87]. The findings revealed that $P$. gingivalis LPS-elicited induction in the acinar cell MMP-9 secretion is accompanied by the enhancement in MT stabilization, while the modulatory influence of peptide hormone, ghrelin, is associated with MT destabilization Further, we found that the changes in MT dynamics induced by the LPS and ghrelin occur through signal-regulated $\alpha$-tubulin phosphorylation on Ser/Tyr [87]. The LPS-induced TLR4 activation and subsequent up-regulation in MMP-9 secretion was reflected in a marked increase in $\operatorname{PKC} \delta$-mediated $\alpha$-tubulin phosphorylation on Ser, while the modulatory influence of ghrelin, an endogenous ligand of the growth hormone secretagogue receptor type 1a (GHS-R1a, a G protein-coupled receptor (GPCR), was manifested in by SFK-PTKs-dependent phosphorylation of $\alpha$-tubulin on Tyr (Figure 4). Moreover, the changes in MT dynamics, conferred by the LPS and ghrelin, were found to affect the Golgi localization of GTP-Arf1 and the recruitment and activation of PKD2.

As the elevated levels of MMP-9 are found in gingival tissue of patients with periodontitis, the above findings suggest that factors affecting MT dynamics and 


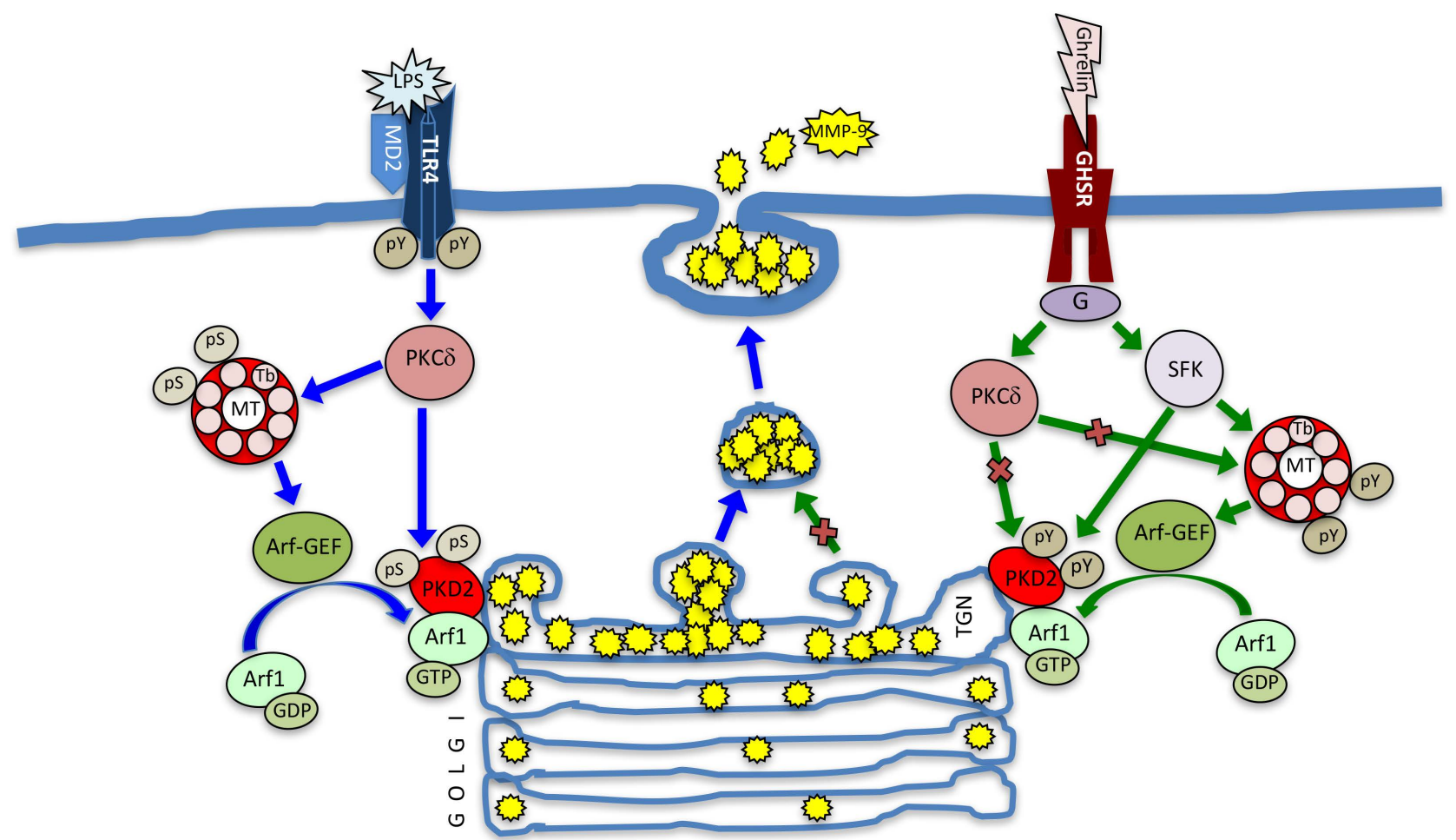

Figure 4. Schematic illustration of the role of $\alpha$-tubulin phosphorylation and the changes in MT dynamics in modulation of salivary gland acinar cell MMP-9 secretion in response to $P$. gingivalis LPS and ghrelin. Engagement of TLR4 by the LPS triggers PKC $\delta$-dependent $\alpha$-tubulin phosphorylation on Ser and the enhancement in MT stabilization, that leads to the up-regulation in Arf-GEF-mediated translocation of Arf1-GTP to the Golgi, and promotes the recruitment and activation of PKD2. The activated PKD2, by acting on the TGN-localized substrates, affects the vesicle fission events and thus enhances the secretion of MMP-9. Ligation by ghrelin of GHS-R1a, on the other hand, leads to SFK-PTKs-mediated phosphorylation of $\alpha$-tubulin on Tyr that maintains MT dynamics at its normal regulatory level. G heterotrimeric G protein, GEF guanine nucleotide exchange factor, MT microtubule, Tb tubulin, pS phosphoserine, pY phosphotyrosine.

MMP-9 secretion present a tempting target for the therapeutic intervention in the treatment of chronic periodontitis. An interesting application of this new type of therapeutic intervention was reported recently the use of spleen tyrosine kinase (Syk) inhibitors to affect MT stability in order to enhance ovarian cell susceptibility to paclitaxel [80].

\section{Conclusions}

MMP-9 is a highly glycosylated zinc-dependent endopeptidase implicated in a wide range of inflammatory and neoplastic diseases, including oral cancer, Sjogren's syndrome, rheumatoid arthritis, and chronic periodontitis, a persistent oral mucosal inflammation attributed primarily to infection by periodontopathic oral pathogen, $P$. gingivalis [5] [6] [7]. Moreover, the elevated levels of MMP-9 in serum of periodontal disease patients have been directly linked to the increase in risk of autoimmune and cardiovascular diseases [5] [9] [35] [88] [89]. Hence, revealing the insights of the signaling cascades involved in the regulation of MMP-9 expression could provide new and innovative options for the treatment of chronic periodontitis and systemic diseases caused by oral infection with $P$. 
gingivalis.

Based on the presented literature data, it is evident that the induction by $P$. gingivalis in oral mucosal release and activation of MMP-9 relays primarily on its key endotoxin, LPS, engagement of TLR4 and the ensuing activation of MAPK signaling cascades. Indeed, the LPS-induced and TLR4-mediated up-regulation in ERK and p38 activation has been demonstrated to trigger the pathway of GEF-regulated Rac-1-GTP membrane translocation that promotes membrane localization of $\mathrm{p} 38$, while ERK was shown to be involved in $\mathrm{CPLA}_{2}$ phosphorylation, its recruitment to the Rac1/p38 complex, and up-regulation in the activation. Apparently, the activated $\mathrm{CPLA}_{2}$ by acting on membrane phospholipids affects the membrane fusion events that result in the induction in MMP-9 release. The role of Rac1/p38 complex in $\mathrm{cPLA}_{2}$ activation is not yet totally clear. There are, however, indications that the complex may be required for the enhancement in $\mathrm{CPLA}_{2}$ activation by $\mathrm{p} 38$-mediated phosphorylation of the phospholipase on the additional Ser sites.

Moreover, it has been established that $P$. gingivalis LPS-elicited induction in MMP-9 secretion, as well as the modulatory influence of peptide hormone, ghrelin, occurs at the level of MMP-9 processing between ER and Golgi, and requires the involvement of factors that control secretory cargo processing, including Arf1 and PKD2. Interestingly, the recent findings indicate that the LPS-induced upregulation in MMP-9 secretion occurs in concert with the changes in stability dynamics of the major cytoskeleton polymeric structures, MTs, which are known to influence the secretory cargo processing and sorting by affecting the Golgi localization of Arf1 and the recruitment and activation of PKD2.

While LPS-induced changes in MT stability dynamics are known to arise through a range of posttranslational modifications of the tubulin subunit of MT, including acetylation and phosphorylation, the $P$. gingivalis LPS-elicited upregulation in MT stability and MMP-9 secretion has been clearly linked to the increase in $\alpha$-tubulin acetylation, while MT destabilizing pharmacological agents were shown to exert the inhibitory effect on the LPS-induced MMP-9 secretion. Interestingly, the modulatory influence of ghrelin on $P$. gingivalis LPS-elicited increase in MMP-9 is associated with MT destabilization. Further dissection of the changes in MT stability dynamics associated with tubulin phosphorylation indicates that the up-regulation in MMP-9 secretion by the LPS is reflected in PKC $\delta$-mediated $\alpha$-tubulin phosphorylation on Ser, while the modulatory effect of ghrelin on MMP-9 secretion is manifested by SFK-PTKs-dependent phosphorylation of $\alpha$-tubulin on Tyr. Therefore, the development of new pharmacological agents selectively targeting MT stability dynamics as well as the processing of MMP-9 between ER and Golgi could provide additional arsenal of therapeutic agents of value in the treatment of chronic periodontitis.

\section{References}

[1] Bodet, C., Chandad, E. and Grenier, D. (2007) Pathogenic Potential of Porphyromonas gingivalis, Treponema denticola and Tannerella forsythia, the Red Bacterial 
Complex Associated with Periodontitis. Pathologie Biologie, 55, 154-162. https://doi.org/10.1016/j.patbio.2006.07.045

[2] Eloe-Fadrosh, E.A. and Rosko, D.A. (2013) The Human Microbiome: From Symbiosis to Pathogenesis. Annual Review of Medicine, 64, 145-163.

https://doi.org/10.1146/annurev-med-010312-133513

[3] How, K.Y., Song, K.P. and Chan, K.G. (2016) Porphyromonas gingivalis: An Overview of Periodontopathic Pathogen Below the Gum Line. Frontiers of Microbiology, 7, Article 53. https://doi.org/10.3389/fmicb.2016.00053

[4] Mysak, J., Pdzimek, S., Sommerova, P., Lyuya-Mi, Y., Bartova, J., Janatova, T., Prochazkova, J. and Duskova, J. (2014) Porphyromonas gingivalis: Major Periodontophatic Pathogen Overview. Journal of Immunology Research, 2014, Article ID: 476068. https://doi.org/10.1155/2014/476068

[5] Li, X., Koltveit, K.M., Tronstad, L. and Olsen, I. (2000) Systemic Diseases Caused by Oral Infection. Clinical Microbiology Reviews, 13, 547-558. https://doi.org/10.1128/CMR.13.4.547-558.2000

[6] Mehta, A. (2015) Risk Factors Associated with Periodontal Diseases and Their Clinical Considerations. International Journal of Contemporary Dental Medical Reviews, 1-15.

[7] Hayashi, S., Yamada, H., Fukui, M., Ito, H.O. and Sata, M. (2015) Correlation between Arteriosclerosis and Periodontal Condition Assessed by Lactoferrin and a1-Antitrypsin Levels in Gingival Crevicular Fluid. International Heart Journal, 56, 639-643. https://doi.org/10.1536/ihj.15-218

[8] Koziel, J., Mydel, P. and Potempa, J. (2014) The Link between Periodontal Disease and Rheumatoid Arthritis: An Updated Review. Current Rheumatology Reports, 16, 408. https://doi.org/10.1007/s11926-014-0408-9

[9] Miklus, T.R., Payne, J.B., Thiele, G.M., et al. (2014) Periodontitis and Porphyromonas gingivalis in Patients with Rheumatoid Arthritis. Arthritis and Rheumatology, 66, 1090-1100. https://doi.org/10.1002/art.38348

[10] Inaba, H., Sugita, H., Kuboniwa, M., et al. (2014) Porphyromonas gingivalis Promotes Invasion of Oral Squamous Cell Carcinoma through Induction of ProMMP-9 and Its Activation. Cell Microbiology, 16, 131-145.

https://doi.org/10.1111/cmi.12211

[11] Katz, J., Onate, M.D., Pauley, K.M., Bhattacharyya, I. and Cha, S. (2011) Presence of Porphyromonas gingivalis in Gingival Squamous Cell Carcinoma. International Journal of Oral Sciences, 3, 209-215. https://doi.org/10.4248/IJOS11075

[12] Datta, H.K., Ng, W.F., Walker, J.A., Tuck, S.P. and Varanasi, S.S. (2008) The Cell Biology of Bone Metabolism. Journal of Clinical Pathology, 61, 577-587. https://doi.org/10.1136/jcp.2007.048868

[13] Amano, A. (2007) Disruption of Epithelial Barrier and Impairment of Cellular Function by Porphyromonas gingivalis. Frontiers in Bioscience, 12, 3965-3974. https://doi.org/10.2741/2363

[14] Nagano, K., Hasegawa, Y., Yoshida, T. and Yoshimura, F. (2015) A Major Fimbrilin Variant of Mfa1 Fimbriae in Porphyromonas gingivalis. Journal of Dental Research, 94, 1143-1148. https://doi.org/10.1177/0022034515588275

[15] Bostanci, N. and Belibasakis, G.N. (2012) Porphyromonas gingivalis. An Invasive and Evasive Opportunistic Oral Pathogen. FEMS Microbiology Letters, 333, 1-9. https://doi.org/10.1111/j.1574-6968.2012.02579.x

[16] Kristoffersen, A.K., Solli, S.J., Nguyen, T.D. and Ensersen, M. (2015) Association of 
the $\operatorname{rgp} B$ Gigipain Genotype to the Major Fimbriae (fimA) Genetype in Clinical Isolates of the Periodontal Pathogen Porphyromonas gingivalis. Journal of Oral Microbiology, 7, Article ID: 29124. https://doi.org/10.3402/jom.v7.29124

[17] DeLeon-Pannell, K.Y., de Castro Bras, L.E. and Lindsey, M.L. (2013) Circulating Porphyromonas gingivalis Lipopolysaccharide Resets Cardiac Homeostasis in Mice through Matrix Metalloproteinase-9-Dependent Mechanism. Physiological Reports, 1, e00079. https://doi.org/10.1002/phy2.79

[18] Slomiany, B.L. and Slomiany, A. (2016) Role of Rac1/p38 and ERK-Dependent Cytosolic Phospholipase $\mathrm{A}_{2}$ Activation in Porphyromonas gingivalis-Evoked Induction in Matrix Metalloproteinase-9 (MMP-9) Release by Salivary Gland Cells. Journal of Biosciences and Medicines, 4, 68-79. https://doi.org/10.4236/jbm.2016.44010

[19] Sochalska, M. and Potempa, J. (2017) Manipulation of Neutrophils by Porphyromonas gingivalis in the Development of Periodontitis. Frontiers in Cellular and Infection Microbiology, 7, 197. https://doi.org/10.3389/fcimb.2017.00197

[20] Slomiany, B.L., Murty, V.L.N., Piotrowski, J., Liau, Y.H. and Slomiany, A. (1993) Glycosulfatase Activity of Porphyromonas gingivalis a Bacterium Associated with Periodontal Disease. Biochemistry and Molecular Biology International, 29, 973-980.

[21] Wang, P.L. and Ohura, K. (2002) Porphyromonas gingivalis Lipopolysaccharide Signaling in Gingival Fibroblasts-CD14 and Toll-Like Receptors. Critical Reviews in Oral Biology and Medicine, 13, 132-142. https://doi.org/10.1177/154411130201300204

[22] Slomiany, B.L. and Slomiany, A. (2003) Activation of Peroxisome Proliferator-Activated Receptor $\gamma$ Impedes Porphyromonas gingivalis Lipopolysaccharide Interference with Salivary Mucin Synthesis through Phosphatidylinositol 3-Kinase/ ERK Pathway. Journal of Physiology and Pharmacology, 54, 3-15.

[23] Park, B.S. and Lee, J.O. (2013) Recognition of Lipopolysaccharide Pattern by TLR4 Complexes. Experimental \& Molecular Medicine, 45, e66.

[24] Amith, S.R., Abdulkhalek, S. and Szewczuk, M.R. (2016) Role of Glycosylation in Toll-Like Receptor Activation and Pro-Inflammatory Responses. In: Weiderschain, G., Ed., Glycobiology and Human Diseases, CRC, Boca Raton, FL, 165-184.

[25] Slomiany, B.L. and Slomiany, A. (2017) Role of LPS-Elicited Signaling in Triggering Gastric Mucosal Inflammatory Responses to H. pylori: Modulatory Effect of Ghrelin. Inflammopharmacology, 25, 415-429. https://doi.org/10.1007/s10787-017-0360-1

[26] Smith, S.M. (2014) Role of Toll-Like Receptors in Helicobacter pylori Infection and Immunity. World Journal of Gastrointestinal Physiology, 5, 133-146. https://doi.org/10.4291/wjgp.v5.i3.133

[27] Coats, S.R., Jones, J.W., Do, C.T., Braham, P.M., Bainbridge, B.W., To, T.T., Goodlett, D.R., Ernst, R.K. and Darveau, R.P. (2009) Human Tool-Like Receptor 4 Responses to P. gingivalis Are Regulated by Lipid A 1- and 4'-Phosphatase Activities. Cellular Microbiology, 11, 1587-1599. https://doi.org/10.1111/j.1462-5822.2009.01349.x

[28] Trent, M.S., Stead, C.M., Tran, A.X. and Hankins, J.V. (2006) Diversity of Endotoxin and Its Impact on Pathogenesis. Journal of Endotoxin Research, 12, 205-223.

[29] Slomiany, B.L. and Slomiany, A. (2005) Role of Modulation of Porphyromonas gingivalis Lipopolysaccharide-Induced Up-Regulation of Endothelin-1 in Salivary Gland Acinar Cells. IUBMB Life, 57, 591-595. https://doi.org/10.1080/15216540500215598

[30] Slomiany, B.L. and Slomiany, A. (2010) Suppression by Ghrelin of Porphyromonas 
gingivalis-Induced Constitutive Nitric Oxide Synthase S-nitrosylation and Apoptosis in Salivary Gland Acinar Cells. Journal of Signal Transduction, 2010, Article ID: 643642. https://doi.org/10.1155/2010/643642

[31] Slomiany, B.L. and Slomiany, A. (2011) Cyclooxygenase-2 S-Nitrosylation in Salivary Gland Acinar Cell Inflammatory Responses to Porphyromonas gingivalis: Modulatory Effect of Ghrelin. Advances in Bioscience and Biotechnology, 2, 434-442. https://doi.org/10.4236/abb.2011.26064

[32] Slomiany, B.L. and Slomiany, A. (2015) Porphyromonas gingivalis-Stimulated TACE Activation for TGF- $\alpha$ Ectodomain Shedding and EGFR Transactivation in Salivary Gland Cells Requires Rac1-Dependent p38 MAPK Membrane Localization. Journal of Biosciences and Medicines, 3, 42-53. https://doi.org/10.4236/jbm.2015.311005

[33] Slomiany, B.L. and Slomiany, A. (2018) Proinflammatory Signaling Cascades of Periodontopathic Oral Pathogen Porphyromonas gingivalis. Journal of Biosciences and Medicines, 6, 63-88. https://doi.org/10.4236/jbm.2018.65009

[34] Vandooren, J., Van den Steen, P.E. and Opdenakker, G. (2013) Biochemistry and Molecular Biology of Gelatinase B or Matrix Metalloproteinase-9 (MMP-9): The Next Decade. Critical Reviews in Biochemistry and Molecular Biology, 48, 222-272. https://doi.org/10.3109/10409238.2013.770819

[35] Ramm, M., Sherr, Y. and Shoenfeld, Y. (2006) Matrix Metalloproteinase-9 and Autoimmune Diseases. Journal of Clinical Immunology, 26, 299-307. https://doi.org/10.1007/s10875-006-9022-6

[36] Perez, P., Kwon, Y.J., Alliende, C., et al. (2005) Increased Acinar Damage of Salivary Glands of Patients with Sjogren's Syndrome is Paralleled by Simultaneous Imbalance of Metalloproteinase-3/Tissue Inhibitor of Metalloproteinase-1 and Matrix Metalloproteinase-9/Tissue Inhibitor of Metalloproteinases-1 Ratios. Arthritis \& Rheumatism, 50, 2751-2760. https://doi.org/10.1002/art.21265

[37] Vilen, S.T., Salo, T., Sorsa, T. and Nyberg, P. (2013) Fluctuating Roles of Matrix Metalloproteinase-9 in Oral Squamous Cell Carcinoma. The Scientific World Journal, 2013, Article ID: 920595. https://doi.org/10.1155/2013/920595

[38] Ejeil, A.L., Igondio-Tchen, S., Ghomrasseni, S., Pellat, B., Godeau, G. and Gogly, B. (2003) Expression of Matrix Metalloproteinases (MMPs) and Tissue Inhibitors of Metalloproteinases (TIMPs) in Healthy and Diseased Human Gingiva. Journal of Periodontology, 74, 188-195. https://doi.org/10.1902/jop.2003.74.2.188

[39] Paulusova, V., Laco, J., Drizhal, I. and Slezak, R. (2012) Expression of Matrix Metalloproteinase-9 in Patients with Oral Lichen Planus. Acta Medica (Hradec Karlove), 55, 23-26. https://doi.org/10.14712/18059694.2015.70

[40] Claveau I., Mostefaoui, Y. and Rouabhia, M. (2004) Basement Membrane Protein and Matrix Metalloproteinase Deregulation in Engineered Human Oral Mucosa Following Infection with Candida albicans. Matrix Biology, 23, 477-486. https://doi.org/10.1016/j.matbio.2004.08.006

[41] Colombo, A.P., Boches, S.K. and Cotton, S.L. (2009) Comparisons of Subgingival Microbial Profiles of Refractory Periodontitis, Severe Periodontitis, and Periodontal Health Using the Human Oral Microbe Identification Microarray. Journal of Periodontology, 80, 1421-1432. https://doi.org/10.1902/jop.2009.090185

[42] Woo, C.H., Lim, J.H. and Kim, J.H. (2004) Lipopolysaccharide Induces Matrix Metalloproteinase-9 Expression via Mitochondrial Reactive Oxygen Species-p38 Kinase-Activator Protein-1Pathway in Raw 264.7 Cells. Journal of Immunology, 173, 6973-6980. https://doi.org/10.4049/jimmunol.173.11.6973

[43] Binker, M.G., Binker-Cosen, A., Richards, D., Oliver, B. and Coswn-Binker, L.I. 
(2009) LPS-stimulated MUC5AC Production Involves Rac-1-Dependent MMP-9 secretion and Activation in NCI-H292 Cells. Biochemical and Biophysical Research Communications, 386, 124-129. https://doi.org/10.1016/j.bbrc.2009.05.136

[44] Jotwani, R., Eswaran, S.V.K., Moonga, S. and Cutler, C.W. (2010) MMP-9/TIMP-1 Imbalance Induced in Human Dendritic Cells by Porphyromonas gingivalis. FEMS Immunology and Medical Microbiology, 58, 314-321. https://doi.org/10.1111/j.1574-695X.2009.00637.x

[45] Rautelin, H.I., Oksanen, A.M., Veijola, L.I., et al. (2009) Enhanced Systemic Metalloproteinase Response in Helicobacter pylori Gastritis. Annals of Medicine, 41, 208-215. https://doi.org/10.1080/07853890802482452

[46] Kubben, F.J.G.M., Sier, C.F.M., Schram, M., et al. (2007) Eradication of Helicobacter pylori Infection Favourably Affects Altered Gastric Mucosal MMP-9 Levels. Helicobacter, 12, 498-504. https://doi.org/10.1111/j.1523-5378.2007.00527.x

[47] Mori, N., Sato, H., Hayashibara, T., et al. (2003) Helicobacter pylori Induces Matrix Metalloproteinase-9 through Activation of Nuclear Factor Kappa B. Gastroenterology, 124, 983-992. https://doi.org/10.1053/gast.2003.50152

[48] Cuadrado, A. and Nebreda, A.R. (2010) Mechanism and Function of p38 MAPK Signaling. Biochemical Journal, 429, 403-417. https://doi.org/10.1042/BJ20100323

[49] Xu, P. and Derynck, R. (2010) Direct Activation of TACE-Mediated Ectodomain Shedding by p38 MAP Kinase Regulates EGF Receptor-Dependent Cell Proliferation. Molecular Cell, 37, 551-566. https://doi.org/10.1016/j.molcel.2010.01.034

[50] Slomiany, B.L. and Slomiany, A. (2006) Leptin Modulates the Detrimental Effect of Porphyromonas gingivalis Lipopolysaccharide-Induced Cytosolic Phospholipase $\mathrm{A}_{2}$ Activation on Salivary Mucin Synthesis via ERK-Signal Transduction. Inflammopharmacology, 14, 250-255. https://doi.org/10.1007/s10787-006-1525-5

[51] Kong, L. and Ge, B.X. (2008) MyD88-Independent Activation of a Novel Actin-Cdc42 /Rac Pathway Is Required for Toll-Like Receptor-Stimulated Phagocytosis. Cell Research, 18, 745-755. https://doi.org/10.1038/cr.2008.65

[52] Yao, H.Y., Chen, L., Wang, J., et al. (2011) Inhibition of Rac Activity Alleviates Lipopolysaccharide-Induced Acute Pulmonary Injury in Mice. Biochimica et Biophysica Acta, 1810, 666-674. https://doi.org/10.1016/j.bbagen.2011.03.020

[53] Brown, W.J., Chambers, K. and Doody, A. (2003) Phospholipase $\mathrm{A}_{2}\left(\mathrm{PLA}_{2}\right)$ Enzymes in Membrane Trafficking: Mediators of Membrane Shape and Function. Traffic, 4, 214-221. https://doi.org/10.1034/j.1600-0854.2003.00078.x

[54] You, H.J., Woo, C.H., Choi, E.Y., et al. (2005) Roles of Rac and p38 Kinase in the Activation of Cytosolic Phospholipase $\mathrm{A}_{2}$ in Response to PMA. Biochemical Journal, 388, 527-535. https://doi.org/10.1042/BJ20041614

[55] Evans, J.H., Gerber, S.H., Murray, D. and Leslie, C.C. (2004) The Calcium Binding Loops of the Cytosolic Phospholipase $\mathrm{A}_{2}$ C2 Domain Specifically Targeting to Golgi and ER in Live Cells. Molecular Biology of the Cell, 15, 371-383.

https://doi.org/10.1091/mbc.e03-05-0338

[56] Hanania, R., Sun, H.S., Xu, K., et al. (2012) Classically Activated Macrophages Use Stable Microtubules for Matrix Metalloproteinase-9 (MMP-9) Secretion. Journal of Biological Chemistry, 287, 8468-8483. https://doi.org/10.1074/jbc.M111.290676

[57] Van den Steen, P.E., Van Aelst, I., Hvidberg, V., et al. (2006) The Hemopexin and O-Glycosylated Domains Tune Gelatinase B/MMP-9 Bioavailability via Inhibition and Binding to Cargo Receptors. Journal of Biological Chemistry, 281, 18626-18637. https://doi.org/10.1074/jbc.M512308200

[58] Rozengurt, E. (2011) Protein Kinase D Signaling: Multiple Biological Functions in 
Health and Disease. Physiology, 26, 23-33.

https://doi.org/10.1152/physiol.00037.2010

[59] Bonnemaison, M.L., Eipper, B.A. and Mains, R.E. (2013) Role of Adaptor Proteins in Secretory Granule Biogenesis and Maturation. Frontiers in Endocrinology, 4, 101. https://doi.org/10.3389/fendo.2013.00101

[60] Bourgoin, S.G. and El Azreq, M.A. (2012) Small Inhibitors of ADP-Ribosylation Factor Activation and Function in Mammalian Cells. World Journal of Pharmacology, 1, 55-64. https://doi.org/10.5497/wjp.v1.i4.55

[61] Donaldson, J.G. and Jackson, C.L. (2011) ARF Family G Proteins and Their Regulators: Roles in Membrane Transport, Development and Disease. Nature Reviews Molecular Cell Biology, 12, 362-375. https://doi.org/10.1038/nrm3117

[62] Cherfils, J. and Zeghouf, M. (2013) Regulation of Small GTPases by GEFs, GAPs, and GDIs. Physiological Reviews, 93, 269-309. https://doi.org/10.1152/physrev.00003.2012

[63] Pusapati, G.V., Krndija, D., Armacki, M., von Wichert, G., et al. (2010) Role of the Second Cysteine-Rich Domain and Pro275 in Protein Kinase D2 Interaction with ADP-Ribosylation Factor 1, Trans-Golgi Network Recruitment, and Protein Transport. Molecular Biology of the Cell, 21, 1011-1022. https://doi.org/10.1091/mbc.e09-09-0814

[64] Wille, C., Kohler, C., Armacki, M., et al. (2014) Protein Kinase D2 Induces Invasion of Pancreatic Cancer Cells by Regulating Matrix Metalloproteinases. Molecular Biology of the Cell, 25, 324-336. https://doi.org/10.1091/mbc.e13-06-0334

[65] Eiseler, T., Wille, C., Koehler, C., Illing, A. and Seufferlein, T. (2016) Protein Kinase D2 Assembles a Multiprotein Complex at the Trans-Gologi Network to Regulate Matrix Metalloproteinase Secretion. Journal of Biological Chemistry, 291, 462-477. https://doi.org/10.1074/jbc.M115.673582

[66] Waldron, R.T. and Rosengurt, E. (2003) Protein Kinase C Phosphorylates Protein Kinase D Activation Loop Ser ${ }^{744}$ and $\mathrm{Ser}^{748}$ and Releases Autoinhibition by the Pleckstrin Homology Domain. Journal of Biological Chemistry, 278, 154-163. https://doi.org/10.1074/jbc.M208075200

[67] Ivison, S.M., Graham, N.R., Bernales, C.Q., et al. (2007) Protein Kinase D Interaction with TLR5 Is Required for Inflammatory Signal in Response to Bacterial Flagellin. Journal of Immunology, 178, 5735-5743.

https://doi.org/10.4049/jimmunol.178.9.5735

[68] Lodeiro, M., Alen, B.O., Mosteiro, C.S., et al. (2011) The SHP-1 Protein Tyrosine Phosphatase Negatively Modulates Akt Signaling in the Ghrelin/GHSR1a System. Molecular Biology of the Cell, 22, 4182-4191. https://doi.org/10.1091/mbc.e11-04-0373

[69] Slomiany, B.L. and Slomiany, A. (2014) Modulation of Gastric Mucosal Inflammatory Responses to Helicobacter pylori via Ghrelin-Induced Protein Kinase C $\delta$ Tyrosine Phosphorylation. Inflammopharmacology, 22, 251-262. https://doi.org/10.1007/s10787-014-0206-Z

[70] Kojima, M., Hosoda, H., Date, Y., et al. (1999) Ghrelin Is a Growth-Hormone-Releasing Acetylated Peptide from Stomach. Nature, 402, 656-660. https://doi.org/10.1038/45230

[71] Groschl, M., Topf, H.G., Bohlender, J., et al. (2005) Identification of Ghrelin in Human Saliva: Production by Salivary Glands and Potential Role in Proliferation of Oral Keratinocytes. Clinical Chemistry, 51, 997-1006. https://doi.org/10.1373/clinchem.2004.040667 
[72] Slomiany, B.L. and Slomiany, A. (2015) Porphyromonas gingivalis-Induced GEF Dock180 Activation by Src/PKC $\delta$-Dependent Phosphorylation Mediates PLC $\gamma 2$ Amplification in Salivary Gland Acinar Cells: Effect of Ghrelin. Journal of Biosciences and Medicines, 3, 66-77. https://doi.org/10.4236/jbm.2015.37008

[73] Huang, C.X., Yuan, M.J., Huang, H., et al. (2009) Ghrelin Inhibits Post-Infarct Myocardial Remodeling and Improves Cardiac Function through Anti-Inflammatory Effect. Peptides, 30, 2286-2291. https://doi.org/10.1016/j.peptides.2009.09.004

[74] Slomiany, B.L. and Slomiany, A. (2016) Role of $\alpha$-Tubulin Acetylation and Protein Kinase D2 Ser/Tyr Phosphorylation in Modulation by Ghrelin of Porphyromonas gingivalis-Induced Enhancement in Matrix Metalloproteinase-9 (MMP-9) Secretion by Salivary Gland Cells. Journal of Biosciences and Medicines, 4, 82-94.

https://doi.org/10.4236/jbm.2016.47009

[75] Goode, B.L., Drubin, D.G. and Barnes, G. (2000) Functional Cooperation between the Microtubule and Actin in Cytoskeletons. Current Opinions in Cell Biology, 12, 63-71. https://doi.org/10.1016/S0955-0674(99)00058-7

[76] Schnaeker, E.F., Ossig, R., Ludwig, T., et al. (2004) Microtubule-Dependent Matrix Metalloproteinase-2/Matrix Metalloproteinase-9 Exocytosis: Prerequisite in Human Melanoma Cell Invasion. Cancer Research, 64, 8924-8931.

[77] Gu, S., Liu, Y., Zhu, B., et al. (2016) Loss of $\alpha$-Tubulin Acetylation Is Associated with TGF- $\beta$-Induced Epithelial-Mesenchymal Transition. Journal of Biological Chemistry, 291, 5396-5405. https://doi.org/10.1074/jbc.M115.713123

[78] Howes, S.C., Alushin, G.M., Shida, T., Nachury, M.V. and Nogales, E. (2014) Effects of Tubulin Acetylation and Tubulin Acetyltransferase Binding on Microtubule Structure. Molecular Biology of the Cell, 25, 257-266. https://doi.org/10.1091/mbc.e13-07-0387

[79] Sbai, O., Ould-Yahoui, A., Ferhart, I., et al. (2010) Differential Vesicular Distribution and Trafficking of MMP-2, MMP-9, and Their Inhibitors in Astrocytes. Glia, 58, 344-366.

[80] Yu, Y., Gaillard, S., Phillip, J.M., et al. (2015) Inhibition of Spleen Tyrosine Kinase Potentiates Paclitaxel-Induced Cytotoxicity in Ovarian Cancer Cells by Stabilizing Microtubules. Cancer Cell, 28, 82-96. https://doi.org/10.1016/j.ccell.2015.05.009

[81] Nirschl, J.J., Magiera, M.M., Lazarus, J.E., Janke, C. and Holzbaur, E.L.F. (2016) $\alpha$-Tubulin Tyrosination and CLIP-170 Phosphorylation Regulate the Initiation of Dynein-Driven Transport in Neurons. Cell Reports, 14, 2637-2652. https://doi.org/10.1016/j.celrep.2016.02.046

[82] Jordan, M.A. and Wilson, L. (2004) Microtubules as a Target for Anticancer Drugs. Nature Reviews, 4, 253-265. https://doi.org/10.1038/nrc1317

[83] Fourest-Lieuvin, A., Peris, L., Gache, V., et al. (2006) Microtubule Regulation in Mitosis: Tubulin Phosphorylation by the Cyclin-Dependent Kinase Cdk1. Molecular Biology of the Cell, 17, 1041-1050. https://doi.org/10.1091/mbc.e05-07-0621

[84] De, S., Tsimounis, A., Chen, X. and Rotenberg, S.A. (2014) Phosphorylation of $\alpha$-Tubulin by Protein Kinase C Stimulates Microtubule Dynamics in Human Breast Cells. Cytoskeleton, 71, 252-272. https://doi.org/10.1002/cm.21167

[85] Slomiany, B.L. and Slomiany, A. (2017) Helicobacter pylori-Induced Changes in Microtubule Dynamics Conferred by $\alpha$-Tubulin Phosphorylation on Ser/Tyr Mediate Gastric Mucosal Secretion of Matrix Metalloproteinase-9 (MMP-9) and Its Modulation by Ghrelin. Inflammopharmacology, 24, 197-205.

https://doi.org/10.1007/s10787-016-0278-z

[86] Slomiany, B.L. and Slomiany, A. (2018) Role of Protein Kinase C $\delta$-Mediated Spleen 
Tyrosine Kinase (Syk) Phosphorylation on Ser in the Amplification of Oral Mucosal Inflammatory Responses to Porphyromonas gingivalis. Journal of Biosciences and Medicines, 6, 70-85. https://doi.org/10.4236/jbm.2018.63005

[87] Slomiany, B.L. and Slomiany, A. (2017) Role of Signal-Regulated Changes in Microtubule Stability Dynamics through $\alpha$-Tubulin Phosphorylation on Ser/Tyr in Modulation of Salivary Gland Matrix Metalloproteinase-9 (MMP-9) Secretion in Response to Porphyromonas gingivalis and Ghrelin. Journal of Biosciences and Medicines, 5, 22-38. https://doi.org/10.4236/jbm.2017.52003

[88] Halade, G.V., Jin, Y.F. and Lindsey, M.R. (2013) Matrix Metalloproteinase (MMP)9: A Proximal Biomarker for Cardiac Remodeling and a Distal Biomarker for Inflammation. Pharmacology \& Therapeutics, 139, 32-40. https://doi.org/10.1016/j.pharmthera.2013.03.009

[89] Yabluchanskiy, A., Ma, Y., Iyer, R.P., Hall, M.E. and Lindsey, M.L. (2013) Matrix Metalloproteinase-9: Many Shades of Function in Cardiovascular Disease. Physiology (Bethesda), 28, 391-403. https://doi.org/10.1152/physiol.00029.2013 